\title{
Structural diversity and biological significance of glycosphingolipids in pathogenic and opportunistic fungi
}

\author{
Luciana L. Guimarães ${ }^{1,2}$, Marcos S. Toledo ${ }^{1}$, Felipe A. S. Ferreira ${ }^{1}$, Anita H. Straus ${ }^{1}$ and \\ Helio K. Takahashi ${ }^{1 *}$
}

1 Laboratory of Glycoconjugate Immunochemistry, Department of Biochemistry, Escola Paulista de Medicina, Universidade Federal de São Paulo, São Paulo, Brazil

${ }^{2}$ Laboratory of Natural Products, Department of Pharmaceutical Sciences, Universidade Santa Cecilia, Santos, Brazil

\section{Edited by:}

Eliana Barreto-Bergter, Universidade

Federal do Rio de Janeiro, Brazil

\section{Reviewed by:}

Charlene Kahler, University of Western Australia, Australia

Leonardo Nimrichter, Federal

University of Rio de Janeiro, Brazil

Hua Xie, Meharry Medical College, USA

\section{*Correspondence:}

Helio K. Takahashi, Laboratory of Glycoconjugate Immunochemistry,

Department of Biochemistry, Escola Paulista de Medicina, Universidade

Federal de São Paulo, Ed. J. L.

Prado, Rua Botucatu, 862,

São Paulo, SP 04023-900, Brazil

e-mail: takahashi.bioq@epm.br
Glycosphingolipids (GSLs) are ubiquitous membrane components and have key roles in biological systems, acting as second messengers or modulators of signal transduction by affecting several events, ranging from cell adhesion, cell growth, cell motility, regulation of apoptosis and cell cycle. Over the last 20 years our laboratory and other research groups determined the glycan and ceramide structures of more than 20 GSLs from several pathogenic/opportunistic fungi, using a combination of gas chromatography, mass spectrometry, nuclear magnetic resonance as well as other immunochemical and biochemical techniques. Fungal GSLs can be divided in two major classes: neutral GSLs, galactosyl- and glucosylceramide (GlcCer), and acidic GSLs, the glycosylinositol-phosphorylceramides (GIPCs). Glycosyl structures in fungal GIPCs exhibited significant structural diversity and distinct composition when compared to mammalian GSLs, e.g., the expression of inositol-mannose and inositol-glucosamine cores and the terminal residue of $\beta$-D-galactofuranose which are absent in mammalian cells. Studies performed by our group demonstrated that GIPC (Galf $\beta 6$ [Man $\alpha 3$ ]Man $\alpha 2$ InsPCer) elicited in patients with paracoccidioidomycosis an immune response with production of antibodies directed to the terminal residue of $\beta$-D-galactofuranose. Further studies also showed that inhibition of GlcCer biosynthetic pathways affects fungal colony formation, spore germination and hyphal growth, indicating that enzymes involved in GlcCer biosynthesis may represent promising targets for the therapy of fungal infections. Recently, it was shown that GlcCer and GIPCs are preferentially localized in membrane microdomains and monoclonal antibodies directed to these GSLs interfere in several fungal biological processes such as growth and morphological transition. This review focuses on glycan structures carried on sphingolipids of pathogenic/opportunistic fungi, and aspects of their biological significance are discussed.

Keywords: fungal glycosphingolipids, glucosylceramide, glycosylinositol phosphorylceramides, glycosphingolipid synthesis inhibitors, fungal membrane microdomains

\section{INTRODUCTION}

Glycosphingolipids (GSLs) are ubiquitous membrane components present mostly in the outer leaflet of the plasma membrane with their carbohydrate head groups exposed to the extracellular space, and mainly organized in microdomains by association with sterols and specific proteins.

The glycosphingolipid biosynthesis starts by the action of serine palmitoyltransferase which condensates palmitoyl-CoA with serine forming 3-keto-sphinganine, the keto group is reduced to hydroxyl group thus forming the dihydrosphingosine (also termed sphinganine), at this point, as shown in Figure 1, the dihydrosphingosine may be hydroxylated on the $\mathrm{C} 4$ to form the phytosphingosine (4-hydroxysphinganine). Specific acyl transferases act upon dihydrosphingosine and phytosphingosine forming dihydroceramide or phytoceramide, respectively. In dihydroceramides, a desaturase inserts a double bound at C4 converting it in ceramide (Rhome et al., 2007). In fungi Glc or Gal residues are transferred to ceramide in the assembly of GlcCer or GalCer whereas the more complex glycan moieties of GIPCs are built up on phytoceramide (Takahashi et al., 2009). After glycosylation, GSLs are transported to the outer leaflet of the cell membrane forming membrane microdomains, also as described by Longo et al. (2013), GlcCer may also be found in lipid vesicles in the cell wall, probably being part of the exocytosis transport system.

Studies analyzing the roles of glycosphingolipids (GSLs) in different biological systems have demonstrated their association with: (i) oncogenic transformation; (ii) embryonic development; (iii) control of cell division; (iv) cell adhesion and motility; (v) signal transduction pathways by glycosinapses, e.g., carbohydrate-carbohydrate interactions; and (vi) control of cell phenotype (Hakomori, 2004, 2008). Since the first description of GSLs in fungi (Steiner et al., 1969), numerous studies focused in structural elucidation and biological significance of these GSLs 


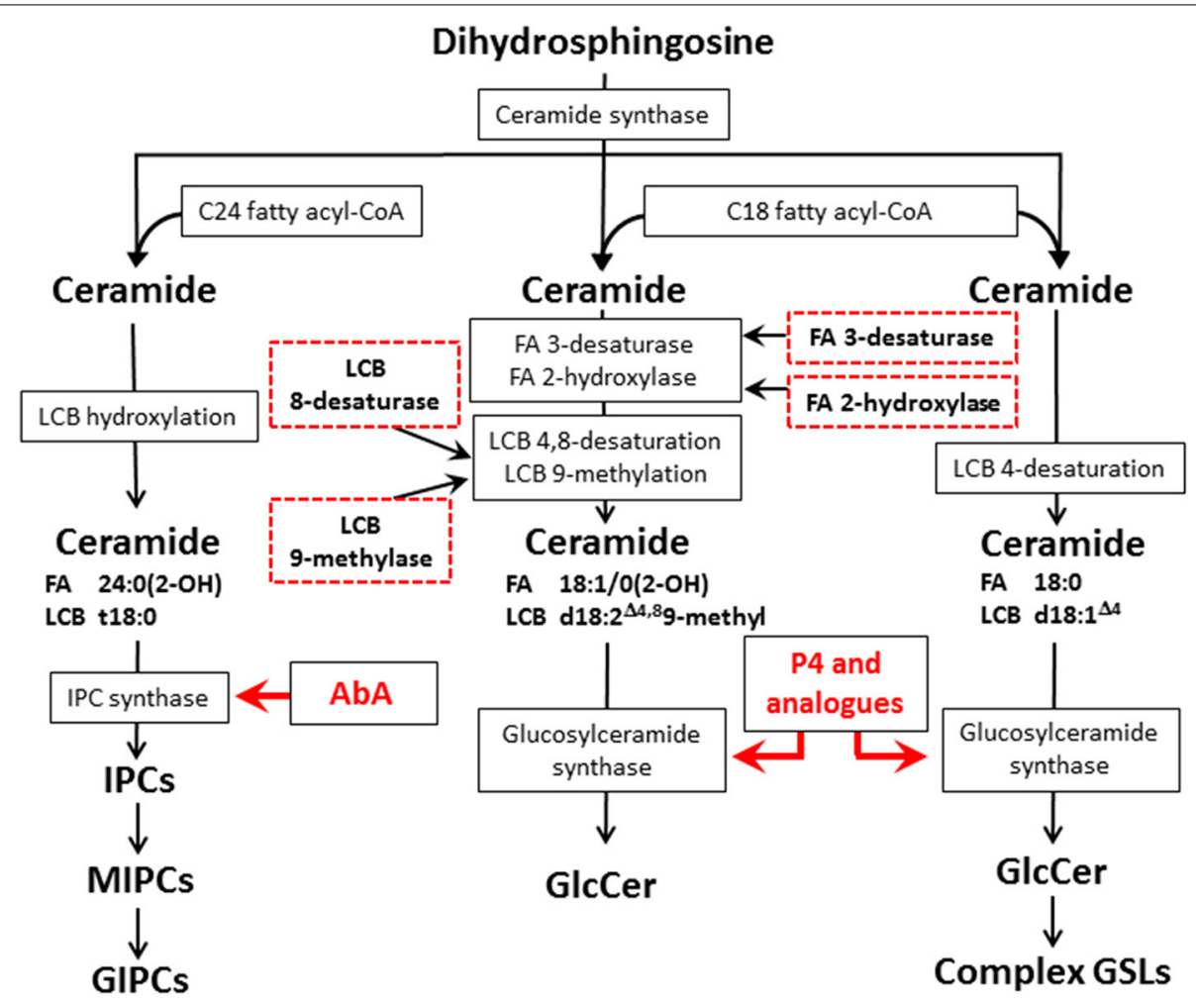

FIGURE 1 | Fungal and mammal GSLs biosynthetic pathways and potential targets for new antifungal therapies. The scheme displays the biosynthetic pathways of fungal GIPCs (left), fungal GlcCer (center), and mammalian GSLs (right). The red arrows indicates the biosynthetic step where the inhibition by AbA and P4/analogs occurs, the red dashed boxes in the glycosphingolipid biosynthetic pathway indicate potential targets for the development of new antifungal therapies. LCB, long chain base; FA, fatty acid; IPC, inositol phosphorylceramide; MIPC, mannosylinositol phosphorylceramide; GIPC, glycosylinositol phosphorylceramide; AbA, Aureobasidin A; P4, D-threo-1-phenyl-2-palmitoyl-3-pyrrolidinopropanol. revealed the importance of these sphingolipids in normal fungal morphogenesis and infectivity. In the last 25 years our lab has made efforts to isolate and analyze the structure of more than 20 new fungal GSLs. In this review we focus on glycan structures carried on sphingolipids of pathogenic/opportunistic fungi and multiple aspects of their biological significance.

\section{STRUCTURAL ELUCIDATION OF GSLS ISOLATED FROM PATHOGENIC FUNGI}

Our laboratory and other research groups have characterized a number of neutral GSLs, glucosyl- and galactosylceramide (GlcCer and GalCer), as well as acidic GSLs- the glycosylinositolphosphorylceramides (GIPCs) from pathogenic/opportunistic fungi, using a combination of high performance thin layer chromatography (HPTLC), gas chromatography/mass spectrometry (GC/MS), single quadrupole mass spectrometry, ${ }^{1} \mathrm{H} /{ }^{13} \mathrm{C}$ nuclear magnetic resonance-Correlation Spectroscopy (COSY), Total Correlation Spectroscopy (TOCSY), Nuclear Overhauser Effect Spectroscopy (NOESY) as well as other immunochemical and biochemical techniques.

As shown in Figure 2 contrasting to mammalian monohexosilceramides, fungal GlcCer and GalCer displays interesting structural peculiarities in their ceramide moiety, such as the presence of (E)- $\Delta^{8}$-unsaturation and a methyl group linked to sphingosine, i.e., (4E,8E)-9-methyl-4,8-sphingadienine as long chain

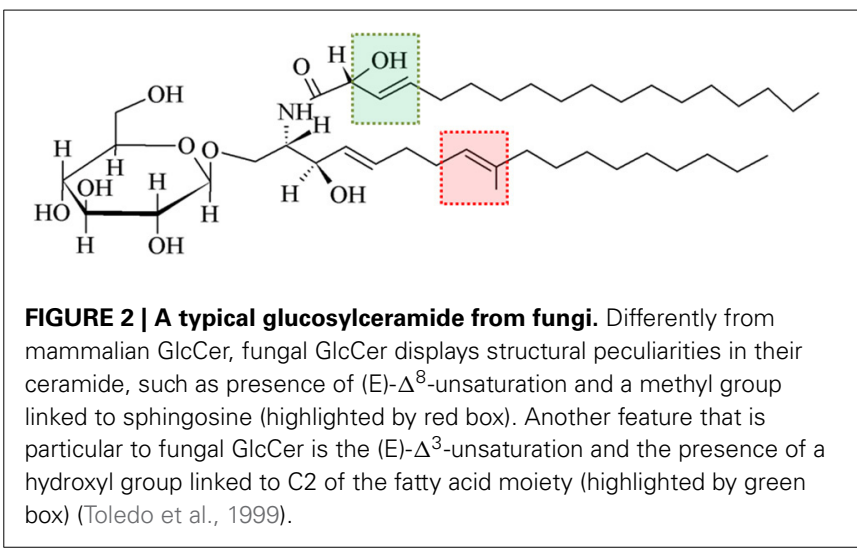

base (d19:2), In Cryptococcus neoformans, the methylation in C9 on the sphingosine backbone of GlcCer is associated with fungi virulence, since the mutation of the sphingolipid C9 methyltransferase gene (SMT1), resulted in loss of more than $80 \%$ of its virulence, when compared to the wild-type and/or the reconstituted strains (Singh et al., 2012).

Another feature that is particular to fungal GlcCer/GalCer is the (E)- $\Delta^{3}$-unsaturation and the presence of a hydroxyl group linked to $\mathrm{C} 2$ of the fatty acid moiety, having either $\mathrm{N}-2^{\prime}$-hydroxyoctadecanoate (h18:0) or the unsaturated form 
N-2'-hydroxy-(E)-3'-octadecenoate (h18:1) [(E)-13-unsaturated fatty acid] (Takahashi et al., 2009). GlcCer from mycelium forms of Paracoccidioides brasiliensis and Histoplasma capsulatum present a higher percentage of unsaturated fatty acids, indicating that the temperature change which induces the transition of mycelium to yeast forms possibly activates a fatty acid desaturase (Toledo et al., 1999, 2001).

Additionally, for yeast forms of Sporothrix schenckii it was observed that the expression of both GlcCer and GalCer was approximately equimolar, while mycelial forms displayed only GlcCer. These differences in neutral GSLs expression suggest that the activation of GalCer synthase may accompanies the mycelium to yeast transition, or, conversely, the suppression of this activity may accompany the yeast to mycelium transition in this fungus (Toledo et al., 2000). Concurrently in two non-dimorphic fungi Aspergillus fumigatus and Aspergillus niger, it was reported the expression of both GlcCer and GalCer, with GalCer bearing a high percentage of unsaturated fatty acid when compared to GlcCer (Villas Boas et al., 1994; Toledo et al., 1999; Levery et al., 2000).

On the other hand, inositol phosphorylceramides (IPCs) and their glycosylated derivatives (GIPCs) are widely distributed among fungal species of the two phyla Ascomycota and Basidiomycota. Fungal GIPCs, display a significant glycosyl structural variation (Barr and Lester, 1984; Barr et al., 1984; Levery et al., 1996, 1998; Loureiro y Penha et al., 2001; Toledo et al., 2007; Suzuki et al., 2008). The glycan structures of fungal GIPCs are built-up on three different "cores": (i) GlcN $\alpha 1$ -

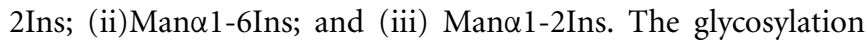
of IPCs seems to occur upon a ceramide mainly composed by t18:0 4-hydroxysphinganine (phytosphingosine) and h24:0 fatty acid (Takahashi et al., 2009). It is worth mentioning that the lipid moiety of these GIPCs when compared to glucosyl- and galactosylceramide displayed different sphingosines, with 4,8diene-9-methyl-sphingo base as precursor for GlcCer/GalCer synthesis, and phytosphingosine for IPC synthesis, suggesting a dichotomy in the biosynthetic pathway of fungal neutral and acidic GSLs (Leipelt et al., 2001). The expression of GIPCs in fungi is summarized in Figure 3.

From an evolutionary perspective, an analysis of GSLs in basal lineages of fungi, such as zygomycetes (James et al., 2006; McLaughlin et al., 2009), revealed that this phylum expresses only neutral GSLs, no inositol-containing sphingolipids were detected (Aoki et al., 2004), differently from higher fungi representatives such as Ascomycota and Basidiomycota where both neutral GSLs and GIPCs are expressed.

Thus, considering the structural diversity of fungal GSLs, studies aiming to investigate the structure-function relationship of these glycoconjugates and their phylogenetic distribution in fungi kingdom may open new perspectives allowing to identify specific targets for new generation of antifungal drugs.

\section{INHIBITION OF GSL BIOSYNTHETIC PATHWAYS AS TARGETS FOR NEW ANTIFUNGAL THERAPIES}

Hence, in order to better understand the importance/biological role of GSLs in different fungi, combined with studies searching for new alternatives for antifungal therapies, a series of studies were conducted in our laboratory to analyze the inhibition effect of key enzymes involved in biosynthetic pathways of fungal GlcCer and GIPCs.

Studies performed with inhibitors of GlcCer synthase, D-threo-1-phenyl-2-palmitoyl-3-pyrrolidinopropanol (P4) and D-threo-3P,4P-ethylenedioxy-P4 (EDO-P4), showed a strong inhibition of germination and hyphal growth, affecting also fungal colony formation of $A$. fumigatus and A. nidulans (Levery et al., 2002). Similar results were observed when $P$. brasiliensis, $H$. capsulatum, S. schenckii, and C. neoformans were cultivated in the presence of P4 (Takahashi et al., 2009). It is worth mentioning that antimicrobial peptides, such as the plant defensin RsAFP2, also display antifungal activity against Candida isolates by interaction with fungal GlcCer (Tavares et al., 2008; Thevissen et al., 2012; Silva et al., 2014). Further improvement of existing GlcCer synthase inhibitors, based on the active site of the fungal enzyme may confer higher selectivity for these compounds, a key step for a more efficient therapy of fungal infections, with fewer side effects on the patients.

Other approaches may also lead to interesting results in studies regarding GlcCer and its influence in host/pathogen interactions, which consists in the use of GlcCer-deficient mutants ( $\Delta$ gcs1) of pathogenic fungi. As shown by Rittershaus et al. (2006) C. neoformans mutant strain lacking GlcCer was unable to grow in vitro at a neutral/alkaline $\mathrm{pH}$ in the presence of $5 \% \mathrm{CO}_{2}$, a condition that mimics the host extracellular environment, such as in alveolar spaces or in the bloodstream. However, growth of these mutants was similar to wild type at acidic $\mathrm{pH}$, which mimics the host intracellular environment, such as macrophage-phagolysosome. Furthermore, when these GlcCer defective mutants were incubated with J774.16 macrophage-like cells, no differences in intracellular growth of mutant cells were observed in comparison to the wild-type, suggesting that GlcCer does not have a relevant role in $C$. neoformans intracellular development. Considering the fact that in Cryptococcus infections they are predominantly in the extracellular environment, GlcCer may represent a highly relevant molecule associated with virulence of $C$. neoformans.

In the last few years, RNAi technology has been used in silencing genes in Saccharomyces spp and Candida albicans yeasts (Drinnenberg et al., 2009; Moazeni et al., 2012). Also, some specific features of fungal GlcCer may represent potential targets for therapy, e.g., methylation at C9 and desaturation at C8 of sphingosine, hydroxylation at $\mathrm{C} 2$ and desaturation at $\mathrm{C} 3$ of the fatty acid (Figure 1). Using a similar approach, the expression of fungal glucosylceramide synthase (GCS) as well as other enzymes related to this biosynthetic pathway could be reduced. As pointed out in a recent review by Del Poeta et al. (2014) GlcCer may be considered a key molecule in fungal infectivity, therefore, this approach may help to develop new therapeutic strategies based on silencing specific target sequences not present in mammals.

Concerning the other biosynthetic route of GSLs in fungi, the IPC and GIPCs synthesis, the first step is catalyzed by the transfer of a phosphoinositol group from a phosphatidylinositol to a ceramide (or phytoceramide) (Nagiec et al., 1997), which also represents potential target for the development of new antifungal drugs. In cultures of Saccharomyces cerevisiae, the inhibition of IPC synthase by Aureobasidin A (AbA), a highly specific pharmacological inhibitor of IPC synthase (Takesako et al., 1993) 


\section{Phylum: Ascomycota}

Sub-phylum: Saccharomycotina

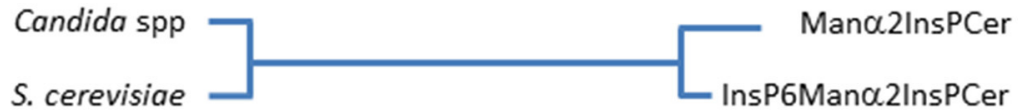

Sub-phylum: Pezizomycotina

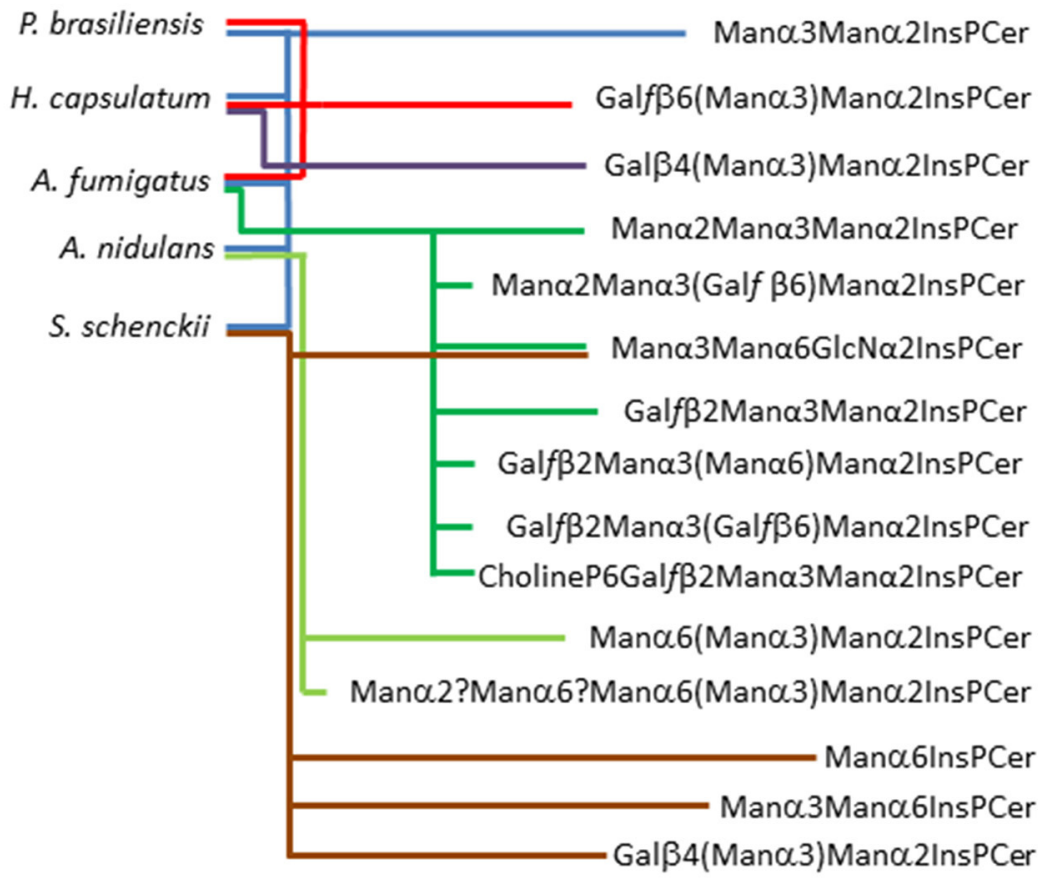

Phylum: Basidiomycota

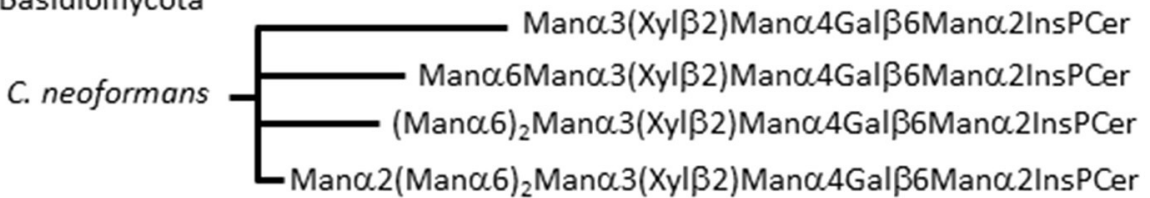

FIGURE 3 | Acidic glycosphingolipids found in fungi according to fungal phylogenetic relationships. The scheme shows two sub-phylum from the Phylum Ascomycota: sub-phylum Saccharomycotina and sub-phylum Pezizomycotina. The former is represented by two of the most studies yeast, Candida spp and S. cerevisiae, their GIPCs were described by Steiner (Steiner et al., 1969), as two basic structures (Man2IPC and IP6Man2IPC). Five fungi are representing the sub-phylum Pezizomycotina, as can be observed they present the most variable repertoire of glycan structures in three possible core: Man $\alpha 2$ IPC, which is present in all five fungi; GlcNa2IPC, which is present in A. fumigatus and S. schenckii; and Man $\alpha 6$ IPC which is present only in S. schenckii (Barr and Lester, 1984; Barr et al., 1984; Toledo et al., 1995, 2000, 2007; Levery et al., 1996, 1998; Loureiro y Penha et al., 2001; Aoki et al., 2004; Suzuki et al., 2008). Phylum Basidiomycota is represented by $C$. neoformans which presents GIPCs with up to eight residues of carbohydrates expressing xylose residues. It is noteworthy that its capsule is rich in xylose (Heise et al., 2002).
(Figure 1), led to the abnormal budding and fungal death (Endo et al., 1997). Takesako et al. (1993) also showed that AbA oral treatment in mice with systemic candidiasis was effective and showed low toxicity for the host. In a more recent experimental approach Tan and Tay (2013) tested the in vitro susceptibility of 92 clinical isolates of various Candida species to AbA. These authors described that planktonic Candida yeasts were more susceptible to AbA than Candida forms present in biofilm $\left(\mathrm{MIC}_{50}\right.$ of 1.0 vs. $8.0 \mu \mathrm{g} . \mathrm{mL}^{-1}$, respectively). In this study it was also demonstrated that AbA inhibited filamentation and lead to short hyphae formation which may have disabled the biofilm development by C. albicans, though biofilm formation and development is a highly complex process which still remain to be fully understood (Shopova et al., 2013; Guimarães and Takahashi, 2014).

Blocking the synthesis of GIPCs with inhibitors also may confer the additional advantage of microbial selectivity considering the fact that this class of GSL is absent in mammalian cells. However, fungi that lack GIPC biosynthetic pathway, such as zygomycetes presented resistance to AbA (Aoki et al., 2004) and they will probably be resistant to others inhibitors of IPC synthase. Therefore, the use of enzymatic inhibitors for one or both GSL biosynthetic pathways must vary according to the fungi, 
allowing the combination of both therapies for most efficient antifungal therapies by blocking GSC and IPC synthase without affecting the biosynthetic pathways of the host.

\section{FUNGAL GSLS AS MODULATORS OF HOST IMMUNE RESPONSE}

Besides the importance of GSLs for normal fungal development, studies performed by our group and other investigators have shown that some fungi elicit immune responses in the infected host. More specifically, it was demonstrated that GIPC Pb-3 (Galf $\beta 6$ [Man $\alpha 3$ ] Man $\alpha 2$ InsPCer) elicited in patients with paracoccidioidomycosis (PCM) an immune response with production of antibodies directed to the terminal residue $\beta$-Dgalactofuranose. Also, several GIPCs from H. capsulatum and A. fumigatus bearing a terminal residue of $\beta \mathrm{Gal} f$ presented crossreactivity with sera of PCM (Barr and Lester, 1984; Toledo et al., 1995, 2007; Bertini et al., 2007). The primary immune response of patients with PCM was associated with IgM production and further switched to IgG1. IgG1 titers decreased after 5 months of antifungal therapy with sulfamethoxazoletrimethoprim accompanied with the decline of the symptoms (Bertini et al., 2007).

Passive immunization with mouse monoclonal antibody directed to Cryptococcus GlcCer was reported to prolong survival of mice infected with C. neoformans (Rodrigues et al., 2007). Conversely, data from our laboratory using a specific monoclonal antibody directed to fungal GlcCer did not show any significant inhibitory effect on P. brasiliensis, H. capsulatum, and S. schenckii colony formation units and fungal growth rate (Toledo et al., 2010). Although promising, the effect of anti-GlcCer antibodies on humans should be carefully assessed in order to fully understand the mechanisms of the modulatory response to anti-GlcCer antibodies as well as to determine their effectiveness as a therapy for different mycosis.

Concerning the cellular immune response against GSLs, experiments were carried out with concanavalin A activated $\mathrm{BALB} / \mathrm{c}$ lymphonode cells and showed that purified preparations of GIPCs from $P$. brasiliensis (Man $\alpha 3$ Man $\alpha 2$ IPC and Galf $\beta 6$ [Man $\alpha 3]$ Man $\alpha 2$ IPC), A. fumigatus (Man $\alpha 2 \operatorname{Man} \alpha 3[\mathrm{Gal} f \beta$ 6]Man $\alpha 2$ IPC) and S. schenckii (Man $\alpha 3$ Man $\alpha 6 \mathrm{GlcN} \alpha 2 \mathrm{IPC}$ ), as well as, GlcCer and GalCer from these three fungi were able to inhibit $\mathrm{T}$ lymphocyte proliferation in vitro in a dose-dependent manner. It was observed an $\mathrm{IC}_{50} \leq 5 \mu \mathrm{M}$ for GIPCs, whereas an $\mathrm{IC}_{50}$ of $20 \mu \mathrm{M}$ was observed for GlcCer and GalCer (Takahashi et al., 2009). Studies performed with GlcCer from A. fumigatus revealed that this GSL was able to activate in vitro mouse and human natural killer T cells (iNKT cells), and to induce airway hyperreactivity in mice (Albacker et al., 2013).

The data above indicate that fungal GSLs, presenting unique monosaccharide sequences and ceramide moieties may influence both humoral and cellular responses and potentially may open new vistas in this field.

\section{FUNGAL GSLS IN MEMBRANE DOMAINS}

Recently it was shown by membrane microdomain isolation protocols that GlcCer and GIPCs are preferentially localized in these membrane domains. Studies performed by our group demonstrated that $\sim 40 \%$ of ergosterol from membranes of $H$. capsulatum is present in membrane microdomain fractions resistant to treatment with non-ionic detergent at $4^{\circ} \mathrm{C}$ (Tagliari et al., 2012). These ergosterol-enriched membrane microdomains showed a peculiar protein distribution and a distinct degree of resistance to treatment with methyl-betacyclodextrin $(\mathrm{m} \beta \mathrm{CD})$, a sterol chelator, suggesting the existence of two populations of membrane microdomains in H. capsulatum yeast forms: Type 1, ergosterol-independent microdomains rich in integrin-like $50 \mathrm{kDa}$ protein and GlcCer and GIPCs, possibly involved in signal transduction; and Type 2, ergosteroldependent microdomains containing Pmalp and the $30 \mathrm{kDa}$ laminin-binding protein (Figure 4). The type 2 microdomains were also shown to be important for infectivity of alveolar macrophage since after the treatment of yeasts forms with $m \beta C D$, the infectivity was reduced by $45 \%$. It is worth mentioning that infectivity of $\mathrm{m} \beta C D$-treated yeasts was completely restored by addition of exogenous ergosterol, but not cholesterol indicating that specifically ergosterol is able to restore the functionality of these fungal membrane domains.

In agreement with these findings, Singh et al. (2012) demonstrated that structural variation in sphingolipids from $C$. neoformans $\Delta s m t 1$ mutant strain (which lacks sphingolipid C9 methyltransferase activity) altered the topography of the membrane lipid affecting fungal plasma membrane rigidity, which was associated with a decrease in the fungal pathogenicity. In the same study, they described that sphingolipid microdomains in C. neoformans wild type are larger and more tightly packed than in $\Delta s m t 1$. Furthermore, these authors also reported extra "soft areas" in the $\Delta s m t 1$ mutant cell membranes, which may lead to a more permeable and more fluid lipid bilayer, resulting in a less rigid conformation of selected sections of the membrane. These data also strongly suggest that the methylated forms of sphingolipids are required for a proper membrane organization associated with fungal virulence.

The dependence of the GSLs organization in plasma membrane for fungal infectivity was studied a using mAbs directed to glycan components of fungal membrane microdomains. We have demonstrated that mAb MEST-3, an IgG2a directed to Manp $\alpha 3$ Manp $\alpha 2$ IPC, interfered on colony formation and morphological transition from yeast to mycelium of $P$. brasiliensis, H. capsulatum and S. schenckii. Similar results were also observed when these fungi where incubated with mAb MEST1 , which reacts with GIPCs presenting terminal residues of $\beta$ D-galactofuranose linked to mannose. A possible explanation for these results could be related to the binding of these two mAbs with cell surface GIPCs thus altering the lipid bilayer organization and hindering the formation of functional membrane microdomains leading to interference in GSLs dependent signaling pathways (Toledo et al., 2010).

Membrane microdomains of host cells were also found to be involved in host cell-pathogen interaction. As demonstrated by our group (Maza et al., 2008) in experiments performed with human lung A549 epithelial cells, the membrane rafts of these cells are involved in adhesion process of $P$. brasiliensis yeast forms, promoting activation of Src-family kinases (SFKs) and extracellular signal-regulated kinase 1/2 (ERK1/2) of these epithelial 


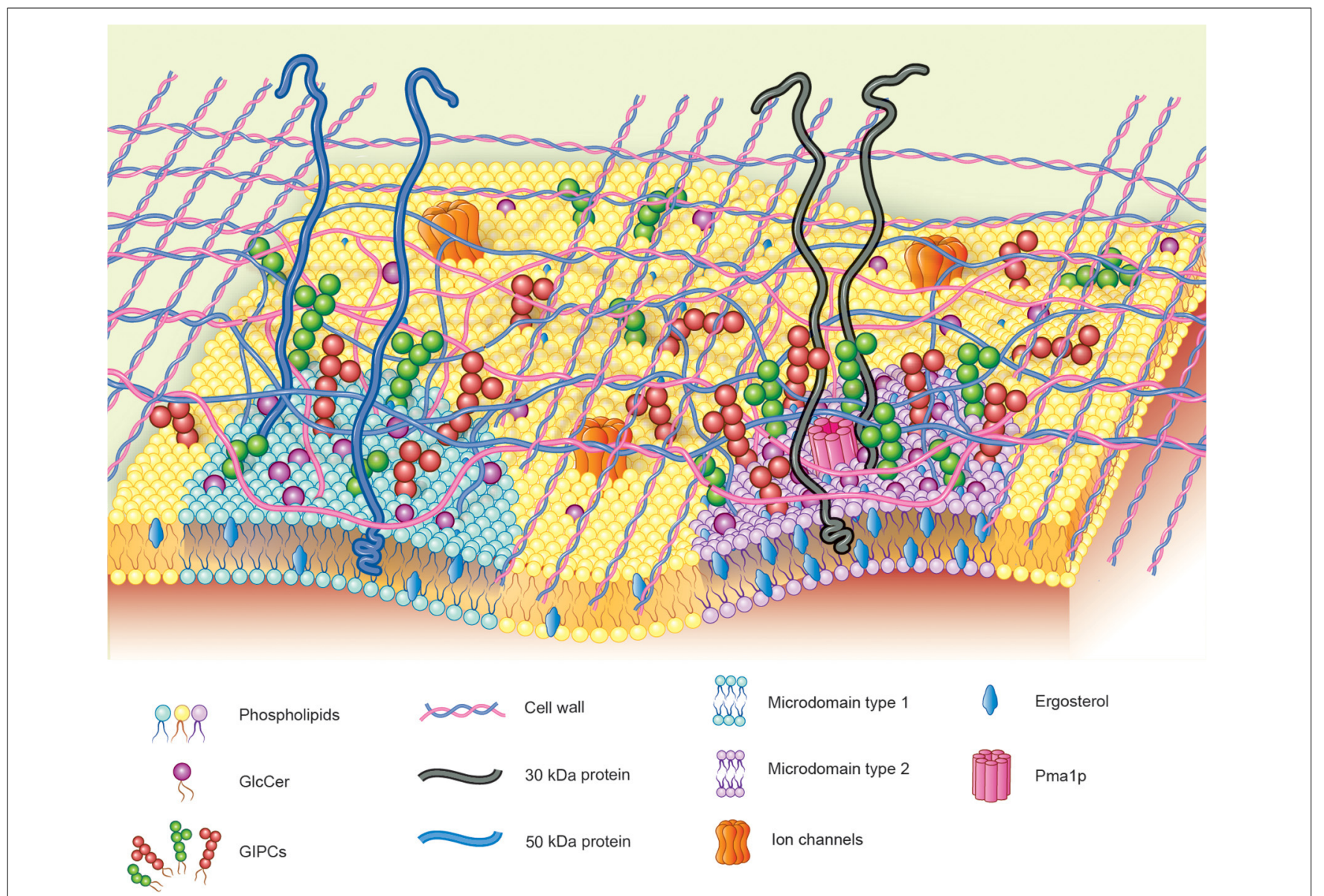

FIGURE 4 | Hypothetical model proposed for fungal membrane microdomains. Two populations of detergent-resistant membrane microdomains are displayed in fungal plasma membrane: Type-1: ergosterol-independent microdomain, rich in integrin-like proteins and GSLs, possibly involved in signal transduction; Type-2: ergosterol-enriched microdomains containing Pma1p and the $30 \mathrm{kDa}$ laminin-binding protein. cells. The activation of epithelial cells SFKs or ERK1/2 might be involved in expression of host inflammatory cytokines and therefore $P$. brasiliensis through its microdomains could be involved in modulation of host immune responses.

The concept of "glycosynapses" introduced by Hakomori (2004) helps to understand the nature and complexity of microdomains/membrane rafts interactions between host-fungal cells. The glycan moiety of fungal GIPCs and GlcCer/GalCer acting in a concerted way with carbohydrate sequences of GSLs/glycoproteins of complementary host cell microdomains (trans-interaction) may intermediate cell-to-cell adhesion with concurrent signal transduction.

\section{CONCLUSIONS AND FUTURE PERSPECTIVES}

Due to its importance in clinical contexts, fungal infections have raised questions regarding its biological process and the key molecules related with the infection maintenance and host cell-pathogen interaction. Considering the structural diversity and the biological roles described for fungal GlcCer and GIPCs, disruption of these biosynthetic pathways may represent an interesting/inviting approach to new vistas for fungal infection therapy. Furthermore, considering the influence of fungal GSLs in host immune response, they may also be considered as fungal biomarkers to detect and identify fungal infections, and as well as follow-up markers of a mycosis at different stages.

Taking these findings together, the next steps would include the elucidation of the biological role of GSLs in the interaction of host-fungal cells during the course of the infection. As shown by Maza et al. (2008) and Tagliari et al. (2012) membrane microdomains of both fungi and host cells play important roles in these interactions, such as observed for fungal adhesion to epithelial cells and fungal infectivity of alveolar macrophages. Additionally, other issues regarding the role of fungal cell wall in the cellular contact must also be elucidated. It is tempting to hypothesize that around the fungal membrane microdomain regions, the glycans of the cell wall could be less tight, leading to a more "coarse" organization, this hypothetical model is tentatively shown in Figure 4. This more loose conformation of the cell wall around the microdomain regions could expose these membrane structures such as: adhesins, glycosphingolipids and glycoproteins allowing the cross-talk of pathogen-host cell through the membrane microdomains.

Cellular membranes are not autonomous cellular structures, since they are linked to intracellular and extracellular networks 
(Nicolson, 2014). One attractive hypothesis is that fungal cell wall could act as biological interface or "conduit" for the information transfer between membrane microdomain and cell wall, perhaps in a glycan-glycan communication and thus mediating the transmission of signals either on localized points or in a network at the cell wall modulating biological events in fungal biology as well as in the fungal infection.

An in-depth knowledge of fungal microdomain interactions in combination with the elucidation of the GSL glycan-dependent signaling pathways and cell wall biosynthesis regulatory steps will certainly provide an integrated view allowing to elaborate a more refined concept of the concerted membrane microdomain-GSL-cell wall involved in key events related to survival and proliferation of pathogenic/opportunistic fungi in the human host.

\section{ACKNOWLEDGMENT}

This work was supported by Fundação de Amparo à Pesquisa do Estado de São Paulo (FAPESP), Conselho Nacional de Desenvolvimento Científico e Tecnológico (CNPq), Coordenação de Aperfeiçoamento de Pessoal de Nível Superior (CAPES).

\section{REFERENCES}

Albacker, L. A., Chaudhary, V., Chang, Y. J., Kim, H. Y., Chuang, Y. T., Pichavant, M., et al. (2013). Invariant natural killer T cells recognize a fungal glycosphingolipid that can induce airway hyperreactivity. Nat. Med. 19, 1297-1304. doi: 10.1038/nm.3321

Aoki, K., Uchiyama, R., Yamauchi, S., Katayama, T., Itonori, S., Sugita, M., et al. (2004). Newly discovered neutral glycosphingolipids in aureobasidin A-resistant zygomycetes: identification of a novel family of Gala-series glycolipids with core Gal alpha 1-6Gal beta 1-6Gal beta sequences. J. Biol. Chem. 279, 32028-32034. doi: 10.1074/jbc.M312918200

Barr, K., Laine, R. A., and Lester, R. L. (1984). Carbohydrate structures of three novel phosphoinositol-containing sphingolipids from the yeast Histoplasma capsulatum. Biochemistry 23, 5589-5596. doi: 10.1021/bi00318a032

Barr, K., and Lester, R. L. (1984). Occurrence of novel antigenic phosphoinositolcontaining sphingolipids in the pathogenic yeast Histoplasma capsulatum. Biochemistry 23, 5581-5588. doi: 10.1021/bi00318a031

Bertini, S., Colombo, A. L., Takahashi, H. K., and Straus, A. H. (2007). Expression of antibodies directed to Paracoccidioides brasiliensis glycosphingolipids during the course of paracoccidioidomycosis treatment. Clin. Vaccine Immunol. 14, 150-156. doi: 10.1128/CVI.00285-06

Del Poeta, M., Nimrichter, L., Rodrigues, M. L., and Luberto, C. (2014). Synthesis and biological properties of fungal glucosylceramide. PLoS Pathog. 10:e1003832. doi: 10.1371/journal.ppat.1003832

Drinnenberg, I. A., Weinberg, D. E., Xie, K. T., Mower, J. P., Wolfe, K. H., Fink, G. R., et al. (2009). RNAi in budding yeast. Science 326, 544-550. doi: $10.1126 /$ science. 1176945

Endo, M., Takesako, K., Kato, I., and Yamaguchi, H. (1997). Fungicidal action of aureobasidin A, a cyclic depsipeptide antifungal antibiotic, against Saccharomyces cerevisiae. Antimicrob. Agents Chemother. 41, 672-676.

Guimarães, L. L., and Takahashi, H. K. (2014). A snapshot of extracellular DNA influence on Aspergillus biofilm. Front. Microbiol. 5:260. doi: 10.3389/fmicb. 2014.00260

Hakomori, S. (2004). Glycosynapses: microdomains controlling carbohydratedependent cell adhesion and signaling. An. Acad. Bras. Cienc. 76, 553-572. doi: 10.1590/S0001-37652004000300010

Hakomori, S. (2008). Structure and function of glycosphingolipids and sphingolipids: recollections and future trends. Biochim. Biophys. Acta. 1780, 325-346. doi: 10.1016/j.bbagen.2007.08.015

Heise, N., Gutierrez, A. L., Mattos, K. A., Jones, C., Wait, R., Previato, J. O., et al. (2002). Molecular analysis of a novel family of complex glycoinositolphosphoryl ceramides from Cryptococcus neoformans: structural differences between encapsulated and acapsular yeast forms. Glycobiology 12, 409-420. doi: 10.1093/ glycob/cwf053
James, T. Y., Kauff, F., Schoch, C. L., Matheny, P. B., Hofstetter, V., Cox, C. J., et al. (2006). Reconstructing the early evolution of Fungi using a six-gene phylogeny. Nature 443, 818-822. doi: 10.1038/nature05110

Leipelt, M., Warnecke, D., Zähringer, U., Ott, C., Müller, F., Hube, B., et al. (2001). Glucosylceramide synthases, a gene family responsible for the biosynthesis of glucosphingolipids in animals, plants, and fungi. J. Biol. Chem. 276, 33621-33629. doi: 10.1074/jbc.M104952200

Levery, S. B., Momany, M., Lindsey, R., Toledo, M. S., Shayman, J. A., Fuller, M., et al. (2002). Disruption of the glucosylceramide biosynthetic pathway in Aspergillus nidulans and Aspergillus fumigatus by inhibitors of UDPGlc: ceramideglucosyltransferase strongly affects spore germination, cell cycle, and hyphal growth. FEBS Lett. 525, 59-64. doi: 10.1016/S0014-5793(02) 03067-3

Levery, S. B., Toledo, M. S., Doong, R. L., Straus, A. H., and Takahashi, H. K. (2000). Comparative analysis of ceramide structural modification found in fungal cerebrosides by electrospray tandem mass spectrometry with low energy collision-induced dissociation of $\mathrm{Li}+$ adductions. Rapid Commun. Mass Spectrom. 14, 551-563. doi: 10.1002/(SICI)1097-0231(20000415)14:7< 551::AID-RCM909>3.0.CO;2-L

Levery, S. B., Toledo, M. S., Straus, A. H., and Takahashi, H. K. (1998). Structure elucidation of sphingolipids from the mycopathogen Paracoccidioides brasiliensis: an immunodominant $\beta$-galactofuranose residue is carried by a novel glycosylinositolphosphorylceramide antigen. Biochemistry 37, 8764-8775. doi: 10.1021/bi9730083

Levery, S. B., Toledo, M. S., Suzuki, E., Salyan, M. E., Hakomori, S., Straus, A. H., et al. (1996). Structural characterization of a new galactofuranosecontaining glycolipid antigen of Paracoccidioides brasiliensis. Biochem. Biophys. Res. Commun. 222, 639-645. doi: 10.1006/bbrc.1996.0796

Longo, L. V., Nakayasu, E. S., Gazos-Lopes, F., Vallejo, M. C., Matsuo, A. L., Almeida, I. C., et al. (2013). Characterization of cell wall lipids from the pathogenic phase of Paracoccidioides brasiliensis cultivated in the presence or absence of human plasma. PLoS ONE 8:e63372. doi: 10.1371/journal.pone.0063372

Loureiro y Penha, C. V., Todeschini, A. R., Lopes-Bezerra, L. M., Wait, R., Jones, C., Mattos, K. A., et al. (2001). Characterization of novel structures of mannosylinositolphosphorylceramides from the yeast forms of Sporothrix schenckii. Eur. J. Biochem. 268, 4243-4250. doi: 10.1046/j.1432-1327.2001.02339.x

Maza, P. K., Straus, A. H., Toledo, M. S., Takahashi, H. K., and Suzuki, E. (2008). Interaction of epithelial cell membrane rafts with Paracoccidioides brasiliensis leads to fungal adhesion and Src-family kinase activation. Microbes Infect. 10, 540-547. doi: 10.1016/j.micinf.2008.02.004

McLaughlin, D. J., Hibbett, D. S., Lutzoni, F., Spatafora, J. W., and Vilgalys, R. (2009). The search for the fungal tree of life. Trends Microbiol. 17, 488-497. doi: 10.1016/j.tim.2009.08.001

Moazeni, M., Khoramizadeh, M. R., Kordbacheh, P., Sepehrizadeh, Z., Zeraati, H., Noorbakhsh, F., et al. (2012). RNA-mediated gene silencing in Candida albicans: inhibition of hyphae formation by use of RNAi technology. Mycopathologia 174, 177-185. doi: 10.1007/s11046-012-9539-6

Nagiec, M. M., Nagiec, E. E., Baltisberger, J. A., Wells, G. B., Lester, R. L., and Dickson, R. C. (1997). Sphingolipid synthesis as a target for antifungal drugs. Complementation of the inositol phosphorylceramide synthase defect in a mutant strain of Saccharomyces cerevisiae by the AUR1 gene. J. Biol. Chem. 272, 9809-9817. doi: 10.1074/jbc.272.15.9809

Nicolson, G. L. (2014). The fluid-mosaic model of membrane structure: still relevant to understanding the structure, function and dynamics of biological membranes after more than 40 years. Biochim. Biophys. Acta 1838, 1451-1466. doi: 10.1016/j.bbamem.2013.10.019

Rhome, R., McQuiston, T., Kechichian, T., Bielawska, A., Hennig, M., Drago, M., et al. (2007). Biosynthesis and immunogenicity of glucosylceramide in Cryptococcus neoformans and other human pathogens. Eukaryotic Cell 6, 1715-1726. doi: 10.1128/EC.00208-07

Rittershaus, P. C., Kechichian, T. B., Allegood, J. C., Merrill, A. H. Jr., Hennig, M., Luberto, C., et al. (2006). Glucosylceramide synthase is an essential regulator of pathogenicity of Cryptococcus neoformans. J. Clin. Invest. 116, 1651-1659. doi: 10.1172/JCI27890

Rodrigues, M. L., Shi, L., Barreto-Bergter, E., Nimrichter, L., Farias, S. E., Rodrigues, E. G., et al. (2007). Monoclonal antibody to fungal glucosylceramide protects mice against lethal Cryptococcus neoformans infection. Clin. Vaccine Immunol. 14, 1372-1376. doi: 10.1128/CVI.00202-07 
Shopova, I., Bruns, S., Thywissen, A., Kniemeyer, O., Brakhage, A. A., and Hillmann, F. (2013). Extrinsic extracellular DNA leads to biofilm formation and colocalizes with matrix polysaccharides in the human pathogenic fungus Aspergillus fumigatus. Front. Microbiol. 4:141. doi: 10.3389/fmicb.2013.00141

Silva, P. M., Gonçalves, S., and Santos, N. (2014). Defensins: antifungal lessons from eukaryotes. Front. Microbiol. 5:97. doi: 10.3389/fmicb.2014.00097

Singh, A., Wang, H., Silva, L. C., Na, C., Prieto, M., Futerman, A. H., et al. (2012). Methylation of glycosylated sphingolipid modulates membrane lipid topography and pathogenicity of Cryptococcus neoformans. Cell. Microbiol. 14, 500-516. doi: 10.1111/j.1462-5822.2011.01735.x

Steiner, S., Smith, S., Waechter, C. J., and Lester, R. L. (1969). Isolation and partial characterization of a major inositol-containing lipid in baker's yeast, mannosyldiinositol, diphosphoryl-ceramide. Proc. Natl. Acad. Sci. U.S.A. 64, 1042-1048. doi: 10.1073/pnas.64.3.1042

Suzuki, E., Tanaka, A. K., Toledo, M. S., Levery, S. B., Straus, A. H., and Takahashi, H. K. (2008). Trypanosomatid and fungal glycolipids and sphingolipids as infectivity factors and potential targets for development of new therapeutic strategies. Biochim. Biophys. Acta 1780, 362-369. doi: 10.1016/j.bbagen.2007.09.009

Tagliari, L., Toledo, M. S., Lacerda, T. G., Suzuki, E., Straus, A. H., and Takahashi, H. K. (2012). Membrane microdomain components of Histoplasma capsulatum yeast forms, and their role in alveolar macrophage infectivity. Biochim. Biophys. Acta 1818, 458-466. doi: 10.1016/j.bbamem.2011.12.008

Takahashi, H. K., Toledo, M. S., Suzuki, E., Tagliari, L., and Straus, A. H. (2009). Current relevance of fungal and trypanosomatid glycolipids and sphingolipids: studies defining structures conspicuously absent in mammals. An. Acad. Bras. Cienc. 81, 477-488. doi: 10.1590/S0001-37652009000300012

Takesako, K., Kuroda, H., Inoue, T., Haruna, F., Yoshikawa, Y., Kato, I., et al. (1993). Biological properties of aureobasidin A, a cyclic depsipeptide antifungal antibiotic. J. Antibiot. (Tokyo) 46, 1414-1420. doi: 10.7164/antibiotics.46.1414

Tan, H. W., and Tay, S. T. (2013). The inhibitory effects of Aureobasidin A on Candida planktonic and biofilm cells. Mycoses 56, 150-156. doi: 10.1111/j.14390507.2012.02225.x

Tavares, P. M., Thevissen, K., Cammue, B. P., François, I. E., Barreto-Bergter, E., Taborda, C. P., et al. (2008). In vitro activity of the antifungal plant defensin RsAFP2 against Candida isolates and its in vivo efficacy in prophylactic murine models of candidiasis. Antimicrob. Agents Chemother. 52, 4522-4525. doi: 10.1128/AAC.00448-08

Thevissen, K., de Mello Tavares, P., Xu, D., Blankenship, J., Vandenbosch, D., Idkowiak-Baldys, J., et al. (2012). The plant defensin RsAFP2 induces cell wall stress, septin mislocalization and accumulation of ceramides in Candida albicans. Mol. Microbiol. 84, 166-180. doi: 10.1111/j.1365-2958.2012.08017.x

Toledo, M. S., Levery, S. B., Bennion, B., Guimaraes, L. L., Castle, S. A., Lindsey, R., et al. (2007). Analysis of glycosylinositolphosphorylceramides expressed by the opportunistic mycopathogen Aspergillusfumigatus. J. Lipid Res. 48, 1801-1824. doi: 10.1194/jlr.M700149-JLR200
Toledo, M. S., Levery, S. B., Straus, A. H., Suzuki, E., Momany, M., Glushka, J., et al. (1999). Characterization of sphingolipids from mycopathogens: factors correlating with expression of 2-hydroxy fatty acyl (E)-13-unsaturation in cerebrosides of Paracoccidioides brasiliensis and Aspergillus fumigatus. Biochemistry 38, 7294-7306. doi: 10.1021/bi982898z

Toledo, M. S., Levery, S. B., Straus, A. H., and Takahashi, H. K. (2000). Dimorphic expression of cerebrosides in the mycopathogen Sporothrix schenckii. J. Lipid Res. 41, 797-806.

Toledo, M. S., Levery, S. B., Suzuki, E., Straus, A. H., and Takahashi, H. K. (2001). Characterization of cerebrosides from the thermally dimorphic mycopathogen Histoplasma capsulatum: expression of 2-hydroxy fatty N- acyl- (E)-Delta(3)unsaturation correlates with the yeast mycelium phase transition. Glycobiology 11, 113-124. doi: 10.1093/glycob/11.2.113

Toledo, M. S., Suzuki, E., Straus, A. H., and Takahashi, H. K. (1995). Glycolipids from Paracoccidioides brasiliensis. Isolation of a galactofuranose-containing glycolipid reactive with sera of patients with paracoccidioidomycosis. J. Med. Vet. Mycol. 33, 247-251. doi: 10.1080/02681219580000501

Toledo, M. S., Tagliari, L., Suzuki, E., Silva, C. M., Straus, A. H., and Takahashi, H. K. (2010). Effect of anti-glycosphingolipid monoclonal antibodies in pathogenic fungal growth and differentiation. Characterization of monoclonal antibody MEST-3 directed to Manpalphal->3Manpalpha1->2IPC. BMC Microbiol. 10:47. doi: 10.1186/1471-2180-10-47

Villas Boas, M. H., Egge, H., Pohlentz, G., Hartmann, R., and Bergter, E. B. (1994). Structural determination of N-20-hydroxyoctadecenoyl-1-O-betaD-glucopyranosyl-9-methyl-4,8-sphingadienine from species of Aspergillus. Chem. Phys. Lipids 70, 11-19. doi: 10.1016/0009-3084(94)90043-4

Conflict of Interest Statement: The authors declare that the research was conducted in the absence of any commercial or financial relationships that could be construed as a potential conflict of interest.

Received: 21 July 2014; accepted: 11 September 2014; published online: 25 September 2014.

Citation: Guimarães LL, Toledo MS, Ferreira FAS, Straus AH and Takahashi HK (2014) Structural diversity and biological significance of glycosphingolipids in pathogenic and opportunistic fungi. Front. Cell. Infect. Microbiol. 4:138. doi: 10.3389/ fimb.2014.00138

This article was submitted to the journal Frontiers in Cellular and Infection Microbiology.

Copyright (c) 2014 Guimarães, Toledo, Ferreira, Straus and Takahashi. This is an open-access article distributed under the terms of the Creative Commons Attribution License (CC BY). The use, distribution or reproduction in other forums is permitted, provided the original author(s) or licensor are credited and that the original publication in this journal is cited, in accordance with accepted academic practice. No use, distribution or reproduction is permitted which does not comply with these terms. 